\title{
National database of patients treated with radical cystectomy provides a current standard and comparison for future technologies
}

\author{
Kazumasa Matsumoto \\ Department of Urology, Kitasato University School of Medicine, Sagamihara, Kanagawa, Japan \\ Correspondence to: Kazumasa Matsumoto. Department of Urology, Kitasato University School of Medicine, Sagamihara, Kanagawa, Japan. \\ Email: kazumasa@cd5.so-net.ne.jp. \\ Provenance: This is a Guest Editorial commissioned by Section Editor Xiao Li (Department of Urology, Jiangsu Cancer Hospital \& Jiangsu Institute \\ of Cancer Research \& Nanjing Medical University Affiliated Cancer Hospital, Nanjing, China). \\ Comment on: Jefferies ER, Cresswell J, McGrath JS, et al. Open radical cystectomy in England: the current standard of care - an analysis of the British \\ Association of Urological Surgeons (BAUS) cystectomy audit and Hospital Episodes Statistics (HES) data. BJU Int 2018. [Epub ahead of print].
}

Submitted Apr 18, 2018. Accepted for publication Apr 23, 2018.

doi: $10.21037 /$ tau.2018.04.22

View this article at: http://dx.doi.org/10.21037/tau.2018.04.22

Both muscle-invasive bladder cancer and high-risk nonmuscle-invasive bladder cancer have been treated using radical cystectomy. Although these aggressive cancers are diseases which depend on radical resection to optimize cancer control, they have been linked to poor prognoses with an estimated 5 -year overall survival rate of approximately $60 \%(1,2)$.

In 2002, the UK National Institute for Health and Care Excellence (NICE) improving outcome guidance (IOG) recommended that radical cystectomies should only be carried out by surgeons handling more than 5 cases per year and in centers where more than 50 pelvic resections, e.g., bladder and prostate, occur. Based on patient records, it is possible to establish whether low-volume surgeons have ceased to perform this procedure and sites assisting fewer patients have referred their patients to an appropriate center nearby. After the IOG recommendation, the median number of cystectomies carried out was 13 patients per surgeon and 23 surgeries per center over a 2-year period (3). In addition, 30- and 90-day mortality rates improved from $2.2 \%$ to $1.3 \%$ and $5.8 \%$ to $2.6 \%$, respectively, between 2003 and 2013. There was, however, no study of the generalized clinical data for open radical cystectomies using the national database, except for other purposes such as billing.

Data entry into the British Association of Urological Surgeons (BAUS) database by surgeons performing radical cystectomy was required in 2013. Combining this BAUS data with the Hospital Episode Statistics (HES) data has allowed comprehensive outcome analysis. The study by Jefferies et al. (3) aimed to make the current standard transparent for patients treated with radical open surgery in England through an examination of records in the 2-year period following the mandate. A total of 2,537 open radical cystectomies were recorded in the BAUS registry and 3,043 in the HES data; based on comparison of patient data, the BAUS database had a capture rate of $83.4 \%$ of all cases, an increase from the $37.1 \%$ capture rate before mandatory data entry (3).

In this study, 30-day mortality was recorded as $1.58 \%$ (40 patients) with 2,497 patients $(98.42 \%$ ) documented as being alive at 30 days and no records leaving this field blank. The 90 -day mortality was recorded as $2.72 \%$ (69 patients), but with only 705 patients $(27.79 \%)$ documented as being alive due to $1,793(69.49 \%)$ patient records having this field left blank. In addition, 710 patients $(28.0 \%)$ had a complication, $1,454(57.3 \%)$ did not and in $373(14.7 \%)$ the field was left blank. High-grade complications (a Clavien-Dindo grade 3 or higher) were reported in 223 patients. Although these findings are definitely a milestone for comparison with other surgical procedures, one possible criticism is that several omissions exist in the data set, especially for 90-day mortality and high-grade complications; these blanks might weaken the results of the study.

Currently, the urological field has adopted the widespread use of a minimally invasive modality. Recent reports have supported the feasibility of laparoscopic and robotassisted surgery for patients with bladder cancer $(1,4,5)$. Because of the high morbidity commonly associated with open procedures, minimally invasive procedures also have potential benefits in terms of perioperative factors related 
to these modalities. A recent systematic review showed that the risk of experiencing postoperative complications was lower with robot-assisted surgery than with open surgery (6). For patients treated with robot-assisted surgery with an extracorporeal conduit, overall 30- and 90-day complication rates were $44 \%$ and $59 \%$, respectively. Highgrade complications at 30 and 90 days were $12 \%$ and $15 \%$. Reported mortality rates at 30 and 90 days were $1.7 \%$ and $1.7 \%$. However, a randomized controlled study comparing these three surgical modalities demonstrated that there were no statistically significant differences among the surgical groups in terms of 30- and 90-day major complications (5). Accumulation of clinical data will reveal the merits and disadvantages of each procedure.

The choice of urinary diversion depends on many factors, including tumor location, clinical stage, age and so on. Most cases in the BAUS database selected ileal conduit for urinary diversion (3). In a German study, the rate of neobladder formation was up to $30 \%$ (7). In a US study, the rate of ileal conduit formation was comparable to this study $(91.0 \%$ and $86.4 \%$, respectively) $(3,8)$. A recent report showed that the share of incontinent urinary diversion in the US remained stable at $93 \%$, while increasing from $63.2 \%$ to $70.8 \%$ in Germany (9). This national database would reveal why the selection of urinary diversion differs between countries and why the clinician would tend to perform ileal conduit.

The surgical concept has moved away from the obsolete principle of one radical procedure. Although the current minimally invasive surgery has demonstrated effective cancer control with minimal impact on quality of life, the management of patients with bladder cancer appears to remain suboptimal. The BAUS cystectomy registry, requiring the entry of data at a national level, is a project unique worldwide. This study provides the current standard for radical open cystectomy in England in order to check their clinical outcomes and to compare future modalities such as minimum invasive surgery and modifications. Continuing data entry will increase the precision and meticulousness of the clinical data and result in better care for the patients.

\section{Acknowledgements}

None.

\section{Footnote}

Conflicts of Interest: The author has no conflicts of interest to declare.

\section{References}

1. Bochner BH, Dalbagni G, Sjoberg DD, et al. Comparing open radical cystectomy and robot-assisted laparoscopic radical cystectomy: A randomized clinical trial. Eur Urol 2015;67:1042-50.

2. Murakami Y, Matsumoto K, Ikeda M, et al. Impact of body mass index on the oncological outcomes of patients with upper and lower urinary tract cancers treated with radical surgery: A multi-institutional retrospective study. Asian Pac J Clin Oncol 2018. [Epub ahead of print].

3. Jefferies ER, Cresswell J, McGrath JS, et al. Open radical cystectomy in England: The current standard of care - an analysis of the BAUS cystectomy audit and HES data. BJU Int 2018. [Epub ahead of print].

4. Matsumoto K, Tabata KI, Hirayama T, et al. Robotassisted laparoscopic radical cystectomy is a safe and effective procedure for patients with bladder cancer compared to laparoscopic and open surgery: Perioperative outcomes of a single-center experience. Asian J Surg 2017. [Epub ahead of print].

5. Khan MS, Gan C, Ahmed K, et al. A Single-centre early phase randomised controlled three-arm trial of open, robotic, and laparoscopic radical cystectomy (CORAL). Eur Urol 2016;69:613-21.

6. Novara G, Catto JW, Wilson T, et al. Systematic review and cumulative analysis of perioperative outcomes and complications after robot-assisted radical cystectomy. Eur Urol 2015;67:376-401.

7. Hautmann RE, Abol-Enein H, Lee CT, et al. Urinary diversion: How experts divert. Urology 2015;85:233-8.

8. Roghmann F, Becker A, Trinh QD, et al. Updated assessment of neobladder utilization and morbidity according to urinary diversion after radical cystectomy: A contemporary USpopulation-based cohort. Can Urol Assoc J 2013;7:E552-60.

9. Groeben C, Koch R, Baunacke M, et al. Urinary diversion after radical cystectomy for bladder cancer: Comparing trends in the US and Germany from 2006 to 2014. Ann Surg Oncol 2018. [Epub ahead of print].

Cite this article as: Matsumoto K. National database of patients treated with radical cystectomy provides a current standard and comparison for future technologies. Transl Androl Urol 2018;7(Suppl 2):S240-S241. doi: 10.21037/tau.2018.04.22 